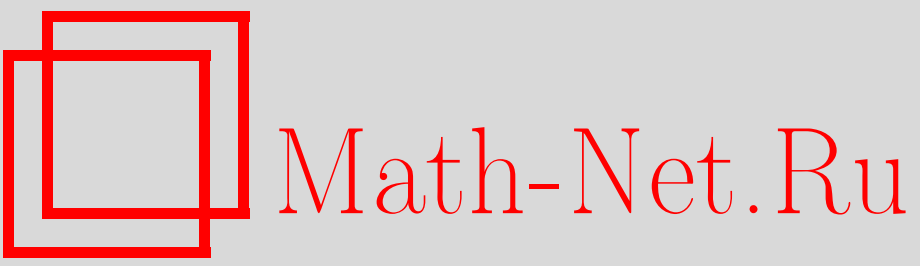

А. А. Логунов, М. А. Мествиришвили, Принцип причинности в полевой теории гравитации, ТМФ, 2001, том 129, номер 1, 153-160

DOI: https://doi.org/10.4213/tmf527

Использование Общероссийского математического портала Math-Net.Ru подразумевает, что вы прочитали и согласны с пользовательским соглашением

http://www.mathnet.ru/rus/agreement

Параметры загрузки:

IP : 54.197 .130 .99

26 апреля 2023 г., 13:17:37 
ТЕОРЕТИЧЕСКАЯ

И МАТЕМАТИЧЕСКАЯ

ФИЗИКА

Том 129, № 1

октябрь, 2001

(C) 2001 г.

А. А. Логунов*, М.А. Мествиришвили*

\section{ПРИНЦИП ПРИЧИННОСТИ В ПОЛЕВОЙ ТЕОРИИ ГРАВИТАЦИИ}

Сформулирован принцип причинности релятивистской теории гравитации, являющийся прямым следствием основных постулатов теории. Установлены необходимые условия, которым должны удовлетворять физические решения уравнений гравитационного поля.

Релятивистская теория гравитации (РТГ) [1], будучи полевой теорией гравитации, основывается на гипотезе, что гравитационное поле, как и все другие поля, развивается в пространстве Минковского, а его источником является такая универсальная сохраняющаяся величина, как тензор энергии-импульса всей материи, включая и гравитационное поле. Такой подход позволяет однозначно (в рамках уравнений второго порядка) построить теорию гравитационного поля как калибровочную теорию.

Полная система гравитационных уравнений РТГ в системе единиц $\hbar=c=G=1$ имеет вид

$$
\begin{gathered}
\gamma^{\alpha \beta} D_{\alpha} D_{\beta} \widetilde{\Phi}^{\mu \nu}+m^{2} \widetilde{\Phi}^{\mu \nu}=16 \pi t^{\mu \nu} \\
D_{\nu} \widetilde{\Phi}^{\mu \nu}=0
\end{gathered}
$$

где $\gamma^{\alpha \beta}$ - метрический тензор пространства Минковского в произвольных координатах, $\widetilde{\Phi}^{\mu \nu}=\sqrt{-\gamma} \Phi^{\mu \nu}-$ плотность тензора гравитационного поля, $D_{\mu}$ - ковариантная производная в пространстве Минковского, $m$ - масса покоя гравитационного поля, $t^{\mu \nu}-$ плотность тензора энергии-импульса всей материи.

Плотность тензора энергии-импульса материи $t^{\mu \nu}$ состоит из плотности тензора энергии-импульса гравитационного поля $t_{\mathrm{g}}^{\mu \nu}$ и плотности тензора энергии-импульса вещества $t_{\mathrm{M}}^{\mu \nu}$. Под вешеством мы подразумеваем все поля материи, за исключением гравитационного поля:

$$
t^{\mu \nu}=t_{\mathrm{g}}^{\mu \nu}+t_{\mathrm{M}}^{\mu \nu}
$$

Взаимодействие гравитационного поля и вещества учитывается в плотности тензора энергии-импульса вешества $t_{\mathrm{M}}^{\mu \nu}$. Гравитационное поле отличается от всех известных

\footnotetext{
* Институт физики высоких энергий, Протвино, Московская обл., Россия
} 
полей тем, что гравитационное взаимодействие затрагивает члены со старшими (вторыми) производными, тогда как все другие поля не входят в члены со вторыми производными. Именно эта особенность гравитационного поля и приводит к возникновению эффективного риманова пространства. Плотность тензора энергии-импульса $t^{\mu \nu}$ согласно Гильберту выражается через скалярную плотность лагранжиана $L$ следующим образом:

где

$$
t^{\mu \nu}=-2 \frac{\delta L}{\delta \gamma_{\mu \nu}},
$$

$$
\frac{\delta L}{\delta \gamma_{\mu \nu}}=\frac{\partial L}{\partial \gamma_{\mu \nu}}-\partial_{\sigma}\left(\frac{\partial L}{\partial \gamma_{\mu \nu, \sigma}}\right), \quad \gamma_{\mu \nu, \sigma}=\frac{\partial \gamma_{\mu \nu}}{\partial x^{\sigma}}
$$

Уравнения (1), (2) можно получить из принципа наименьшего действия, только если плотность лагранжиана взять в виде [1]

$$
L=L_{\mathrm{g}}+L_{\mathrm{M}}\left(\tilde{g}^{\mu \nu}, \Phi_{A}\right),
$$

где

$$
\tilde{g}^{\mu \nu}=\tilde{\gamma}^{\mu \nu}+\widetilde{\Phi}^{\mu \nu}
$$

$\tilde{g}^{\mu \nu}=\sqrt{-g} g^{\mu \nu}, \tilde{\gamma}^{\mu \nu}=\sqrt{-\gamma} \gamma^{\mu \nu}, \Phi_{A}$ - поля вешества. Плотность лагранжиана гравитационного поля $L_{\mathrm{g}}$ в выражении (6) равна [1]

$$
L_{\mathrm{g}}=\frac{1}{16 \pi} \tilde{g}^{\mu \nu}\left(G_{\mu \nu}^{\lambda} G_{\lambda \sigma}^{\sigma}-G_{\mu \sigma}^{\lambda} G_{\nu \lambda}^{\sigma}\right)-\frac{m^{2}}{2}\left(\frac{1}{2} \gamma_{\mu \nu} \tilde{g}^{\mu \nu}-\sqrt{-g}-\sqrt{-\gamma}\right)
$$

где $G_{\mu \nu}^{\lambda}-$ тензор третьего ранга, равный

$$
G_{\mu \nu}^{\lambda}=\Gamma_{\mu \nu}^{\lambda}-\gamma_{\mu \nu}^{\lambda}=\frac{1}{2} g^{\lambda \sigma}\left(D_{\mu} g_{\sigma \nu}+D_{\nu} g_{\sigma \mu}-D_{\sigma} g_{\mu \nu}\right),
$$

$\Gamma_{\mu \nu}^{\lambda}$ - символы Кристоффеля эффективного риманова пространства, $\gamma_{\mu \nu}^{\lambda}-$ символы Кристоффеля пространства Минковского.

Плотности лагранжианов (6) и (8) являются следствием исходных положений теории. Из формулы (6) видно, что движение вешества в пространстве Минковского под действием гравитационного поля сводится к движению вещества в эффективном римановом пространстве. Таким образом и возникает эффективное риманово пространство, которое является прямым следствием гипотезы, что источником гравитационного поля является сохраняющийся тензор энергии-импульса материи.

Поскольку гравитационное поле (например, в инерциальной системе) определяется в одной системе координат, то согласно (7) и эффективное риманово пространство также определяется в одной системе координат. Но это означает, что оно имеет простую топологию. Таким образом, эффективное риманово пространство, возникающее из-за действия гравитационного поля в пространстве Минковского, всегда имеет простую топологию. В общей теории относительности (ОТО) возможны сложные топологии риманова пространства, т.е. для его описания необходим атлас карт. Полевые представления о гравитации весьма жесткие, а поэтому на их основе нельзя получить уравнения ОТО. 
С учетом формул (6) и (8) из принципа наименьшего действия следует, что уравнения (1) и (2) можно записать в форме [1]

$$
\begin{aligned}
R_{\mu \nu}-\frac{m^{2}}{2}\left(g_{\mu \nu}-\gamma_{\mu \nu}\right) & =8 \pi\left(T_{\mu \nu}-\frac{1}{2} g_{\mu \nu} T\right), \\
D_{\nu} \tilde{g}^{\mu \nu} & =0
\end{aligned}
$$

где

$$
T^{\mu \nu}=-2 \frac{\delta L_{\mathrm{M}}}{\delta g_{\mu \nu}}, \quad T_{\mu \nu}=g_{\mu \sigma} g_{\nu \lambda} T^{\sigma \lambda} .
$$

Уравнения (1) и (2), так же как и уравнения (10) и (11), обшековариантны относительно произвольных координатных преобразований и форминвариантны относительно преобразований Лоренца. Последнее означает, что они точно удовлетворяют специальному принципу относительности. Согласно РТГ силы инерции и силы гравитации разделены, они имеют разную природу. Если силы инерции можно обратить в нуль выбором инерциальной системы координат, то силы гравитации нельзя исключить выбором системы координат даже локально. Об этом почти полвека назад Синг писал [2]: “B теории Эйнштейна в зависимости от того, отличен от нуля тензор Римана или равен нулю, гравитационное поле присутствует или отсутствует. Это свойство абсолютно; оно никак не связано с мировой линией какого-то наблюдателя". Рассматривая гравитационное поле как физическое поле в пространстве Минковского, мы должны с необходимостью соблюдать принцип причинности в пространстве Минковского. Суть его заключается в следуюшем: для движушегося пробного тела в пространстве Минковского, какими бы силами оно не вызьвалось, всегда можно выбрать в этом пространстве такую систему координат, в которой это тело будет находиться в состоянии покоя, а следовательно, должно иметь место условие

$$
d \sigma^{2}=\gamma_{00}(x)\left(d x^{0}\right)^{2}>0, \quad \gamma_{00}(x)>0 .
$$

В работе Минковского "Пространство и время" [3], опубликованной в 1909г., это положение было сформулировано в следуюшей форме: "Введем теперь следующую аксиому. Субстаниия, находящаяся в любой мировой точке, всегда при надлежащем определении пространства и времени может быть рассматриваема как находящаяся в покое".

Но учитывая, что в пространстве Минковского под действием гравитационного поля пробное тело движется по геодезической линии эффективного риманова пространства, можно заключить, что должно выполняться также условие

$$
d s^{2}=g_{\mu \nu}(x) d x^{\mu} d x^{\nu}>0
$$

которое в системе координат, где тело покоится, принимает вид

$$
d s^{2}=g_{00}(x)\left(d x^{0}\right)^{2}>0, \quad g_{00}(x)>0 .
$$


Таким образом, при движении пробного тела в пространстве Минковского всегда можно выбрать такую систему координат, в которой это тело находится в покое, но при этом одновременно должны выполняться условия причинности (13) и (15), т.е.

$$
d s^{2}=g_{00}(x)\left(d x^{0}\right)^{2}>0, \quad d \sigma^{2}=\gamma_{00}(x)\left(d x^{0}\right)^{2}>0 .
$$

Эти условия причинности можно записать и в следуюшей форме [1]:

$$
\begin{aligned}
& \gamma_{\mu \nu}(x) U^{\mu} U^{\nu}=0 \\
& g_{\mu \nu}(x) U^{\mu} U^{\nu} \leqslant 0
\end{aligned}
$$

где мы учли также возможность сушествования в природе частиц с нулевой массой покоя, движение которых происходит по изотропным геодезическим линиям. Условия (16), так же как условия (17) и (18), означают, что конус причинности эффективного риманова пространства должен заключаться внутри конуса причинности пространства Минковского. Если бы конус причинности риманова пространства выходил за конус причинности пространства Минковского, то это привело бы к тому, что в пространстве Минковского было бы невозможно выбрать систему координат, в которой пробное тело находилось бы в состоянии покоя. Это означало бы, что трехмерную силу гравитации такого "гравитационного поля" невозможно уравновесить силой инерции, поскольку в этом случае имело бы место неравенство

$$
d \sigma^{2}=\gamma_{\mu \nu}(x) d x^{\mu} d x^{\nu}<0
$$

Отсюда следует, что такое “гравитационное поле" нельзя представить как физическое поле, развивающееся в пространстве Минковского. В силу условий причинности (17) и (18) эффективное риманово пространство будет обладать изотропной и времениподобной геодезической полнотой. Принцип причинности обеспечивает также сушествование во всем римановом пространстве пространственноподобной поверхности, которую каждая непространственноподобная кривая пересекает только один раз, т.е. существует глобальная поверхность Коши, на которой и задаются для той или иной задачи начальные физические условия. При решении уравнений Гильберта-Эйнштейна отбираются только такие решения, для которых в каждой точке пространства имеет место условие

$$
g<0
$$

а также для любого времениподобного вектора $K_{\nu}$ выполняется неравенство

$$
T^{\mu \nu} K_{\mu} K_{\nu} \geqslant 0
$$

причем величина $T^{\mu \nu} K_{\nu}$ для данного вектора $K_{\nu}$ должна образовывать непространственноподобньй вектор.

В РТГ при решении уравнений (10) и (11) необходимо отбирать только такие решения, которые наряду с условиями (20) и (21) удовлетворяют также условиям причинности (17) и (18). Условия причинности не следуют из уравнений, но в этом нет ничего 
странного, поскольку и в электродинамике принцип причинности не является следствием уравнений Максвелла-Лоренца, он вводится дополнительно в форме (13). Из уравнений (10) и (11) следует, что пробное тело движется по геодезической линии эффективного риманова пространства, определяемого уравнением

$$
\frac{d p^{\nu}}{d s}+\Gamma_{\alpha \beta}^{\nu} p^{\alpha} p^{\beta}=0, \quad p^{\nu}=\frac{d x^{\nu}}{d s}, \quad d s^{2}=g_{\mu \nu} d x^{\mu} d x^{\nu}>0
$$

где символ Кристоффеля

$$
\Gamma_{\alpha \beta}^{\nu}=\frac{1}{2} g^{\nu \sigma}\left(\partial_{\alpha} g_{\sigma \beta}+\partial_{\beta} g_{\sigma \alpha}-\partial_{\sigma} g_{\alpha \beta}\right)
$$

Согласно РТГ такое движение не является свободным, поскольку оно осушествляется в пространстве Минковского под действием силы гравитационного поля. В ОТО отсутствует понятие силы гравитации. Синг об этом писал так [2]: "B теории относительности понятие силь гравитации отсутствует, так как гравитационные свойства органически входят в структуру пространства-времени и проявляются в кривизне пространства-времени, т.е. в том, что тензор Римана $R_{\mu \nu \lambda \sigma}$ отличен от нуля".

В РТГ понятие силы гравитации сохранено, поскольку гравитация обязана существованию гравитационного поля в пространстве Минковского. Ниже мы определим эту силу, опираясь на принцип причинности (17) и (18) и следуя [4]. Согласно определению ковариантной производной в пространстве Минковского имеем

$$
\frac{D p^{\nu}}{d s}=\frac{d p^{\nu}}{d s}+\gamma_{\alpha \beta}^{\nu} p^{\alpha} p^{\beta}
$$

Подставляя (22) в (23), находим

$$
\frac{D p^{\nu}}{d s}=-G_{\alpha \beta}^{\nu} p^{\alpha} p^{\beta}
$$

Запишем левую часть соотношения (24) в форме

$$
\frac{D p^{\nu}}{d s}=\left(\frac{d \sigma}{d s}\right)^{2}\left[\frac{D V^{\nu}}{d \sigma}+V^{\nu} \frac{\frac{d^{2} \sigma}{d s^{2}}}{\left(\frac{d \sigma}{d s}\right)^{2}}\right], \quad V^{\nu}=\frac{d x^{\nu}}{d \sigma}
$$

Здесь $V^{\nu}$ - времениподобный 4-вектор скорости в пространстве Минковского, удовлетворяющий условию

$$
\gamma_{\mu \nu} V^{\mu} V^{\nu}=1, \quad d \sigma^{2}>0 .
$$

Подставляя (25) в (24), получим

$$
\frac{D V^{\nu}}{d \sigma}=-G_{\alpha \beta}^{\nu} V^{\alpha} V^{\beta}-V^{\nu} \frac{\frac{d^{2} \sigma}{d s^{2}}}{\left(\frac{d \sigma}{d s}\right)^{2}}
$$


На основании условия (26) имеем

$$
\left(\frac{d \sigma}{d s}\right)^{2}=\gamma_{\alpha \beta} p^{\alpha} p^{\beta}
$$

Дифференцируя это выражение по $d s$, получим

$$
\frac{\frac{d^{2} \sigma}{d s^{2}}}{\left(\frac{d \sigma}{d s}\right)^{2}}=-\gamma_{\lambda \mu} G_{\alpha \beta}^{\mu} V^{\lambda} V^{\alpha} V^{\beta}
$$

Подставляя это выражение в (27), найдем [4]

$$
\frac{D V^{\nu}}{d \sigma}=-G_{\alpha \beta}^{\mu} V^{\alpha} V^{\beta}\left(\delta_{\mu}^{\nu}-V^{\nu} V_{\mu}\right)
$$

Отсюда очевидно, что движение пробного тела в пространстве Минковского происходит под действием 4-вектора силы

$$
F^{\nu}=-G_{\alpha \beta}^{\mu} V^{\alpha} V^{\beta}\left(\delta_{\mu}^{\nu}-V^{\nu} V_{\mu}\right), \quad V_{\mu}=\gamma_{\mu \sigma} V^{\sigma}
$$

Легко убедиться, что

$$
F^{\nu} V_{\nu}=0
$$

Левая часть уравнения (30) по определению равна

$$
\frac{D V^{\nu}}{d \sigma}=\frac{d V^{\nu}}{d \sigma}+\gamma_{\alpha \beta}^{\nu} V^{\alpha} V^{\beta}
$$

Следует особо отметить, что движение пробного тела по геодезической линии эффективного риманова пространства может быть представлено как движение в пространстве Минковского под действием силы $F^{\nu}$, только если одновременно имеют место условия

$$
d s^{2}=g_{\mu \nu} d x^{\mu} d x^{\nu}>0, \quad d \sigma^{2}=\gamma_{\mu \nu} d x^{\mu} d x^{\nu}>0
$$

т.е. вьполняется принцип причинности.

Сила гравитации и тензор кривизны Римана взаимно связаны. Так, если тензор кривизны Римана равен нулю, то в силу уравнений (10) и (11) будет равна нулю и сила гравитации $F^{\nu}$. В том случае, когда тензор кривизны отличен от нуля, сила гравитации также не равна нулю. И наоборот, если сила гравитации $F^{\nu}$ отлична от нуля, то и кривизна Римана не равна нулю. Обрашение в нуль силы гравитации $F^{\nu}$ приводит к равенству нулю тензора кривизны Римана. Благодаря действию силы гравитации $F^{\nu}$ и происходит падение тела в гравитационном поле, т.е. происходит все так, как это имеет место в ньютоновой физике. Более того, все гравитационные эффекты в Солнечной системе (отклонение луча света Солнцем, временное запаздывание радиосигнала, прецессия гироскопа, смешение перигелия Меркурия) вызваны именно действием силы гравитации $F^{\nu}$, а не тензором кривизны Римана, который в Солнечной системе достаточно 
мал. Это объясняется тем, что сила гравитации определяется первыми производными от метрики, тогда как тензор кривизны Римана - вторыми производными. Поскольку сила гравитации $F^{\nu}$ является 4-вектором, то она никогда не может быть обрашена в нуль выбором системы координат. Это и означает, что гравитационное поле, как физическую реальность, нельзя устранить даже локально. Аналогичное утверждение имеет место для любого физического поля. Поскольку движение пробного тела по геодезической линии эффективного риманова пространства не является свободным, то система, падаюшая в гравитационном поле, не является даже локально-инерциальной. Если бы пробное тело было заряженным, то оно излучало бы электромагнитные волны, как движушееся с ускорением. Именно поэтому ускорение в РТГ имеет абсолютный смысл, поскольку сохранено понятие инерциальной системы координат.

В работе [5] отмечается, что в линейном приближении для слабой монохроматической волны принцип причинности (17) и (18) нарушается. Однако это заключение неправильное. Уравнения (10) и (11) в линейном приближении по теории возмушений принимают вид

$$
\begin{gathered}
\gamma^{\alpha \beta} \partial_{\alpha} \partial_{\beta} \Phi^{\mu \nu}+m^{2} \Phi^{\mu \nu}=8 \pi T^{\mu \nu} \\
\partial_{\nu} \Phi^{\mu \nu}=0
\end{gathered}
$$

где $\gamma^{\alpha \beta}=(1,-1,-1,-1)$. В линейном приближении не учитывается взаимодействие гравитационного поля и вешества, а также взаимодействие гравитонов между собой. Согласно уравнениям (35), (36) условие причинности как для гравитационного поля, так и для других полей имеет обычный вид:

$$
d \sigma^{2}=\gamma_{\mu \nu} d x^{\mu} d x^{\nu} \geqslant 0
$$

Отсюда следует, что все физические поля не выходят за рамки конуса пространства Минковского, а поэтому принщип причинности не нарушается и для слабых волн (в том числе, электромагнитных). Согласно уравнениям (35) и (36) движение пробных тел, света и других полей будет происходить по геодезическим линиям пространства Минковского. Следует особо отметить, что система уравнений $(35),(36)$ физически противоречива, поскольку согласно (35) и (36), с одной стороны, имеет место закон сохранения энергии-импульса вещества

$$
\partial_{\nu} T^{\mu \nu}=0
$$

а с другой стороны, возникает излучение гравитационного поля $\Phi^{\mu \nu}$, что с необходимостью приводит к потере энергии-вешества, а это противоречит закону сохранения тензора энергии-импульса вешества (38).

Введение принципа причинности в форме (16) или $(17),(18)$ является не произвольным требованием, а с необходимостью возникает как прямое следствие гипотезы, что гравитационное поле, как и все другие физические поля, развивается в пространстве Минковского. Именно такое представление и реализуется в РТГ, причем метрический тензор пространства Минковского содержится в системе исходных уравнений (10), (11). 
В литературе бытует утверждение, что ОТО можно представить в форме полевой теории с использованием пространства Минковского. Это неправильно. Полевые представления, как мы уже отмечали, с необходимостью приводят к простой топологии эффективного риманова пространства, а также требуют соблюдения принципа причинности в пространстве Минковского. Но все это отсутствует в ОТО, и в этом ее особенность. Использование метрики пространства Минковского в ОТО лишено какого-либо физического смысла и противоречит логике этой теории. Полевые представления огравитации вместе с гипотезой, что сохраняющийся тензор энергии-импульса является источником гравитационного поля, с необходимостью приводят к выводу о существовании массы покоя гравитационного поля, что и нашло отражение в системе уравнений РТГ. Таким образом, предположение о наличии массы покоя гравитационного поля вытекает из следующих достаточно общих положений теории [1]: гравитационное поле является физическим полем, развивающимся в пространстве Минковского подобно другим физическим полям, и источником этого поля является универсальная сохраняющаяся величина - тензор энергии-импульса всей материи, включая гравитационное поле.

Поскольку между электродинамикой Максвелла-Лоренца и РТГ имеется большое сходство по построению, то естественно предположить, как это ранее отмечалось [6], возможность существования массы покоя и у фотона.

Благодарности. Авторы выражают благодарность В. И. Денисову и Ю. В. Чугрееву за ценные обсуждения.

\section{Список литературы}

[1] А. А. Логунов, М. А. Мествиришвили. Релятивистская теория гравитации. М.: Наука, 1989; A. A. Logunov. Relativistic Theory of Gravity. Nova Science Publishers, Inc. V. 215. New York: Commack, 1998; A. А. Логунов. Теория гравитационного поля. М.: Наука, 2000. [2] Дж. Синг. Общая теория относительности. М.: ИЛ, 1963.

[3] Г. Минковский. Пространство и время. В сб.: Принцип относительности. Сост. А. А. Тяпкин. М.: Атомиздат, 1973. С. 171.

[4] N. Rosen. Phys. Rev. 1940. V. 57. P. 147-153.

[5] J. Brian Pitts and W. C. Schieve. Slightly bimetric gravitation. gr-qc/0101058.

[6] А. А. Логунов. Вестн. МГУ. Сер. 3. Физика, астрономия. 1993. Т. 34. № 4. С. 3-19.

Поступила в редакцию 27.III.2001 г. 